Erratum

J Mol Evol (1981) 18:38-46

\title{
Comparative Biosequence Metrics
}

T.F. Smith, M.S. Waterman, and W.M. Fitch

Page 40, lines 1 and 2 of second column should read:

The sum $s+d$, does not equal $n \alpha_{m a x}$ because the prime on $w^{\prime}{ }_{m-n}$ was missed.

The proper conclusion is:

$\mathrm{s}+\mathrm{d}=0.5(\mathrm{~m}+\mathrm{n}) \alpha_{\max }$ which is true whether $\mathrm{m} 3 \mathrm{n}$ or not. 